\title{
The TMD Program at JLab
}

\section{Haiyan Gao*†}

Department of Physics, Duke University, Durham, North Carolina 27708, USA

Duke Kunshan University, Kunshan, Jiangsu 215316, China

E-mail: gao@phy.duke.edu

\section{Tianbo Liu}

Department of Physics, Duke University, Durham, North Carolina 27708, USA

Thomas Jefferson National Accelerator Facility, Newport News, VA 23606, USA

E-mail: liutbejlab.org

\section{Zhiwen Zhao}

Department of Physics, Duke University, Durham, North Carolina 27708, USA

E-mail: zhiwen.zhao@phy.duke.edu

\begin{abstract}
As a part of the general nucleon imaging effort, there have been many efforts to access the transverse momentum dependent parton distributions (TMDs) by using the semi-inclusive deep inelastic scattering (SIDIS) processes. The recently upgraded Continuous Electron Beam Accelerator Facility (CEBAF) at Jefferson Lab (JLab) provides golden opportunities to study them in valence quark region. The TMDs describe the three-dimensional, spin-correlated distributions of quarks and gluons in the nucleon in momentum space. The corresponding SIDIS measurements require high intensity and polarization with large kinematic coverage which will be provided by several different detectors. We present an overview of the planned JLab TMD program in the $12 \mathrm{GeV}$ era.
\end{abstract}

XXVI International Workshop on Deep-Inelastic Scattering and Related Subjects (DIS2018)

16-20 April 2018

Kobe, Japan

\footnotetext{
* Speaker.

${ }^{\dagger}$ This work is supported in part by the U.S. Department of Energy, Office of Science, Office of Nuclear Physics under contract Nos. DE-FG02-03ER41231 and DE-AC05-06OR23177.
} 


\section{TMD Physics}

Nucleons remain among the most mysterious objects that human beings have ever studied. The quantum chromodynamics (QCD), the theory of the strong force in the Standard Model, cannot give a satisfactory description of a nucleon from first principles of QCD because it is not solvable by the commonly used perturbation method at the long distance or low energy limit. Carefully designed experiments therefore play the central role in pushing forward our understanding of the structure of the nucleon.

A general framework to describe the partonic structure of a nucleon is the generalized transverse momentum dependent parton distributions (GTMDs). The Wigner distributions are related to GTMDs via a transverse Fourier transformation. There is no realistic way to measure any of them in experiments directly yet. However, we can access them partially by measuring the generalized parton distributions (GPDs) and transverse momentum dependent parton distributions (TMDs).

TMDs have gained a lot of interest in recent years. Within the TMD factorization, SIDIS structure functions are expressed as convolutions of transverse momentum dependent parton distribution functions (TMD PDFs) and transverse momentum dependent fragmentation functions (TMD FFs). There are eight leading-twist (twist-two) quark TMD PDFs for a nucleon. If the transverse momentum is integrated, three of them, $f_{1}, g_{1 L}$, and $h_{1}$, will reduce to their collinear limits: the unpolarized PDF, helicity distribution, and transversity distribution, while the remaining five will vanish. Hence TMDs, especially the spin-dependent ones, contain much richer information than collinear PDFs, and allow us to access the correlation between quark transverse momentum and quark/nucleon spin.

Among the eight leading-twist TMDs, one highlight is the quark transversity distribution, $h_{1}\left(x, \mathbf{k}_{\perp}^{2}, Q^{2}\right)$, which also has a collinear definition $h_{1}\left(x, Q^{2}\right)$. It is dominated by the valance quark distribution and can be studied by measuring the Collins Target Single Spin Asymmetry. There were extensive experimental studies and global fits to the existing world data in the last few years [1, $2,3]$. We will present some projected impacts of the JLab TMD program on transversity in the later section.

\section{TMD Program at Jefferson Lab $12 \mathrm{GeV}$}

The $12 \mathrm{GeV}$ energy upgrade at JLab provides a unique opportunity to study the quark TMDs, particularly in the valence quark region [4]. A coherent and comprehensive TMD program using SIDIS measurements is planned using the upgraded $12 \mathrm{GeV}$ CEBAF at Jefferson Lab. It takes advantages of the complementary capabilities of different detectors in experimental Halls A, B and C.

\subsection{CLAS12 SIDIS Experiments}

The CLAS12 spectrometer in Hall B with a design similar to the CLAS spectrometer from the $6 \mathrm{GeV}$ era, is a general-purpose detector of charged and neutral particles with momenta above $1 \mathrm{GeV} / \mathrm{c}$, scattering angles greater than 5 degrees, and nearly $2 \pi$ azimuthal angular acceptance. The large acceptance and an order-of-magnitude higher design luminosity of $10^{35} \mathrm{~cm}^{-2} \cdot \mathrm{s}^{-1}$, compared to CLAS spectrometer, makes it ideal for broad surveys of the entire kinematic phase space of the 
SIDIS reaction accessible with an $11 \mathrm{GeV}$ beam. Its planned measurements include unpolarized targets and polarized targets, such as dynamically polarized ammonia $\left(\mathrm{NH}_{3}\right.$ and $\left.\mathrm{ND}_{3}\right)$ and transversely polarized solid HD in the frozen-spin state (HDice). Its unpolarized data are being collected with fully differential mapping of the unpolarized SIDIS cross sections, including hadron multiplicities and azimuthal moments. The polarized experiments will follow and together they will help lay the foundation for the theoretical interpretation of SIDIS data and the global extraction of TMDs in the valence region.

\subsection{HMS\&SHMS SIDIS Experiments}

Hall $\mathrm{C}$ in the $12 \mathrm{GeV}$ era hosts the pre-existing High Momentum Spectrometer (HMS) and the new Super High Momentum Spectrometer (SHMS) with the capablity of reaching more forward scattering angles and a maximum momentum of $11 \mathrm{GeV}$. These small-acceptance, focusing magnetic spectrometers have well-defined acceptances and excellent angular and momentum resolution. The experiments in Hall C plan to measure the unpolarized SIDIS cross section with high precision in well-defined, targeted kinematics. These measurements will provide detailed understanding of the reaction mechanism of the SIDIS process such as the applicability of the factorization, L-T separation, and can be a powerful cross check of the large-acceptance data from detectors like CLAS12.

\subsection{BS\&SBS SIDIS Experiments}

For the polarized SIDIS measurements, it is desired to have large acceptance and high luminosity to maximize kinematic coverage and precision. The experiment which uses the existing BigBite spectrometer and the new Super BigBite Spectrometer (SBS) on a high-pressure transversely polarized ${ }^{3} \mathrm{He}$ target is a big step in that direction. It increases the statistical figure-of-merit for target single spin asymmetry measurements by roughly a factor of 1000 compared to the Hall A $6 \mathrm{GeV}$ SIDIS experiment $[5,6]$ by using the moderate solid angle and large momentum acceptance of SBS in place of the small-acceptance HRSs, and by increasing the design luminosity of the polarized ${ }^{3} \mathrm{He}$ target by roughly a factor of 5 , to a total useful luminosity of about $1.8 \times 10^{37} \mathrm{~cm}^{-2} \cdot \mathrm{s}^{-1}$.

\subsection{SoLID SIDIS Experiments}

To fully utilize the CEBAF upgrade and to maximize its discovery potentials, the Solenoidal Large Intensity Device (SoLID), capable of handling both high luminosities and large acceptance, was proposed and designed for Hall A to satisfy a highly rated and rich scientific program [7]. Among the five approved SoLID experiments, three are SIDIS experiments involving a transversely polarized ${ }^{3} \mathrm{He}$ target, a longitudinally polarized ${ }^{3} \mathrm{He}$ target, and a transversely polarized proton target, all with A ratings. They are designed to measure the single/double spin asymmetries through the SIDIS $\left(e, e^{\prime} \pi^{ \pm}\right)$process with the SoLID spectrometer.

The layout of the SoLID SIDIS setup is shown in Fig. 1. It consists of two sub-groups of detectors at forward angle and large angle. The polar angular coverage is $8^{\circ}-15^{\circ}$ for the forward-angle detector and $15^{\circ}-24^{\circ}$ for the large-angle detector. Both of them have a full azimuthal coverage. The scattered electrons are detected by either the forward-angle or large-angle detectors and the leading charged pions are detected by the forward-angle detector only. Two electromagnetic 


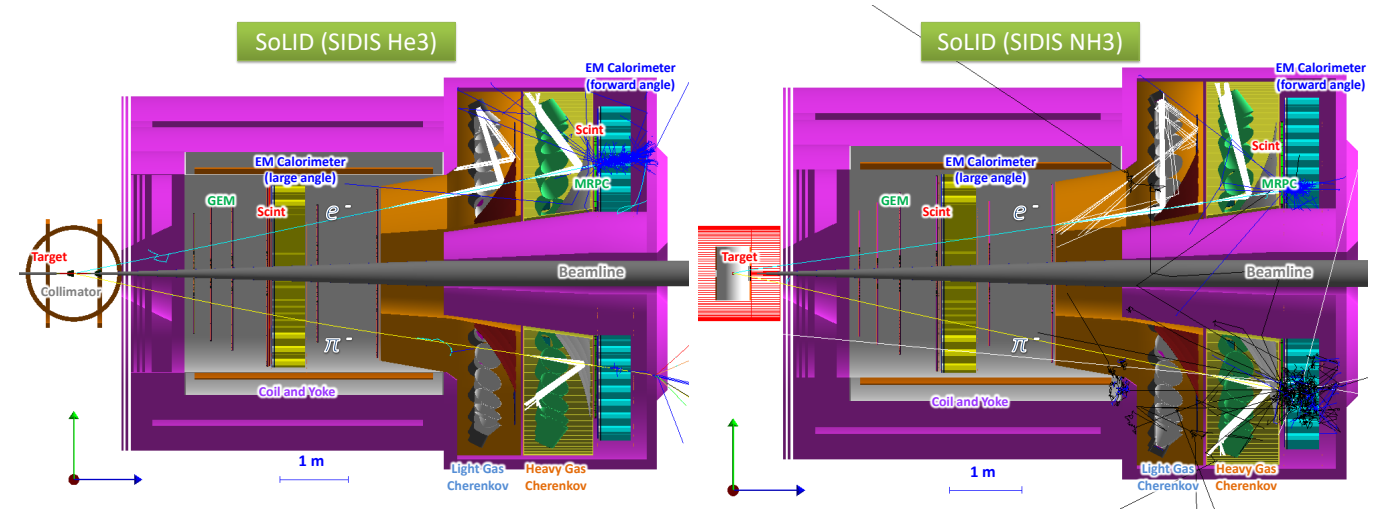

Figure 1: The experimental layout of the SoLID SIDIS setup with the ${ }^{3} \mathrm{He}$ (left) or $\mathrm{NH}_{3}$ (right) target. The scattered electrons are detected by both forward-angle and large-angle detectors. The leading pions are detected by the forward-angle detector only.

calorimeters made of Shashlyk type modules with good resolution and high radiation tolerance are used for electron/pion separation. A $2 \mathrm{~m}$ long light gas Čerenkov counter is installed at the forward angle to further improve the identification of scattered electrons. A $1 \mathrm{~m}$ long heavy gas Čerenkov counter right after the light gas Čerenkov counter can separate kaons and protons from the pions at momenta larger than $2.5 \mathrm{GeV}$. One layer of multi-gap resistive plate chamber is placed after the heavy gas Čerenkov counter to provide timing information and particle identification of hadrons at low momentum $(<2.5 \mathrm{GeV})$, as well as to suppress photon background. One layer of scintillator pad detector is placed in front of the forward angle electromagnetic calorimeter to reject photons. Another layer of scintillator pad detector is placed in front of the large angle electromagnetic calorimeter to reject photons and provide timing information and particle identification of hadrons at low momentum also. Five layers of gas electron multiplier detectors provide tracking for both sub-groups detectors in this high rate environment.

A polarized ${ }^{3} \mathrm{He}$ gas target will be used as an effective polarized neutron target with a $60 \%$ in beam polarization and a fast spin flip. Placed in front of the spectrometer entrance, it can take $15 \mu$ A electron beams to reach the polarized luminosity of $10^{36} \mathrm{~N} \mathrm{~cm}^{-2} \cdot s^{-1}$. To study the SIDIS process on proton, a cryogenic $\mathrm{NH}_{3}$ solid target whose protons are polarized to $70 \%$ will be placed at the same location to take $0.1 \mu$ A electron beams to reach the polarized luminosity $10^{35} \mathrm{~N} \mathrm{~cm}^{-2}$ $\mathrm{s}^{-1}$. All of SoLID sub-systems including the detectors and DAQ system are designed to handle the high luminosity with large acceptance. This is the key advantage of the SoLID SIDIS program which is capable of collecting high statistics data with well controlled systematics.

The expected SoLID SIDIS kinematic coverage includes: i) $0.05<x<0.6$ which comprises the majority of the valence quark region; ii) $0.3<z<0.7$ in which the leading order $x-z$ factorization is expected to hold; iii) maximum pion transverse momentum $P_{T}$ up to $1.6 \mathrm{GeV}$; and iv) $1 \mathrm{GeV}^{2}<Q^{2}<8 \mathrm{GeV}^{2}$. These kinematic coverages can be achieved by combining data with incident electron energies of 11 and $8.8 \mathrm{GeV}$. The distribution of bins in $x-Q^{2}$ plane and the comparison to HERMES and COMPASS bins are shown in Fig. 2 [8]. The SoLID SIDIS experiments plan to extend to a larger $x$ region with $Q^{2}$ coverage comparable with HERMES. The average sta- 
tistical precision of each bin for SoLID is about $1 \%$ consisting of more than 600 bins for $p\left(e, e^{\prime}\right) \pi^{+}$ channel, compared to $37.1 \%$ (relative to the size of the asymmetry) for HERMES consisting of 7 bins in $x$ for the same channel. We have a cut of $W>2.3 \mathrm{GeV}$ to leave the feasibility of usage of low $W$ region for future developments.

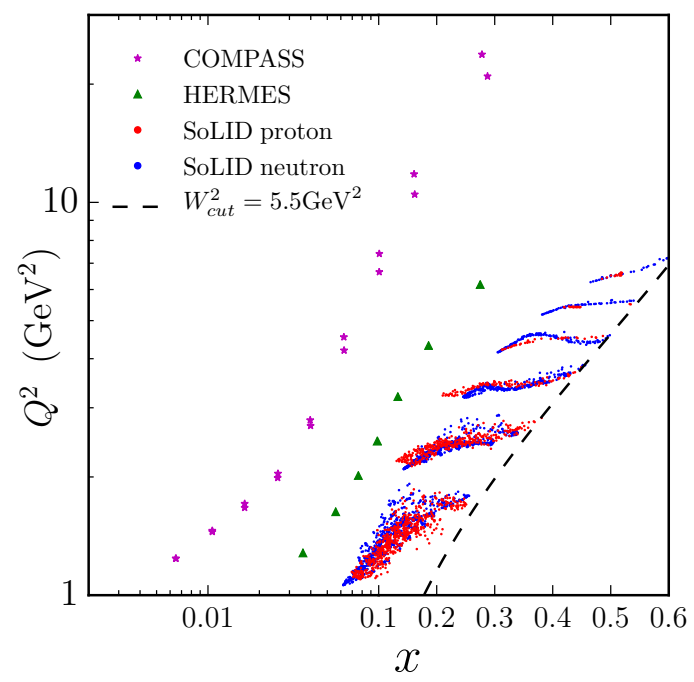

Figure 2: $x-Q^{2}$ kinematical plane of bins for SoLID data compared with HERMES and COMPASS data sets. The figure is from Ref. [8].

The physics impact of SoLID is the precise measurement of the TMDs in the valance region. To compare the improvement on the determination of transversity, we model the transversity distributions with the recent global fit of [1], which includes the TMD evolution effect. We used simulated data for SoLID SIDIS experiments to estimate the impact of the future SoLID data on the transversity distribution of $u$ - and $d$-quarks. Our method follows Bayesian statistics where the new information is added sequentially on top of the prior knowledge without requiring a combined analysis of the old data and the new data by using the Hessian approach.

Our results for $u$ - and $d$-quark transversity distributions at $Q^{2}=2.4 \mathrm{GeV}^{2}$ are presented in Fig. 3 along with results from the global fit to existing data by Ref. [1] (KPSY15). In the upper row of plots, the uncertainties of KPSY15 are given as light shaded bands, while the projected errors after the SoLID data are taken into account are shown as dark shaded bands. To quantify the improvement of adding the future SoLID data, we show, in the bottom row of plots, the ratio of the estimated errors relative to the current errors. The results are shown using only the proton data (left panels), the neutron data (central panels), and the combination of the proton and the neutron data sets (right panels). While the absolute error bands can differ depending on the error analysis, the ratio of the errors is almost independent of the error analysis. Notice that the maximal improvements are attained in the region covered by the SoLID data $0.05<x<0.6$ where the errors will be reduced by approximate $90 \%$, i.e. one order of magnitude, for both $u$ - and $d$-quark transversities. The impact decreases outside of this region as expected.

\section{Summary}

TMD is a critical part of understanding of nucleon structure and QCD in general. The TMD 

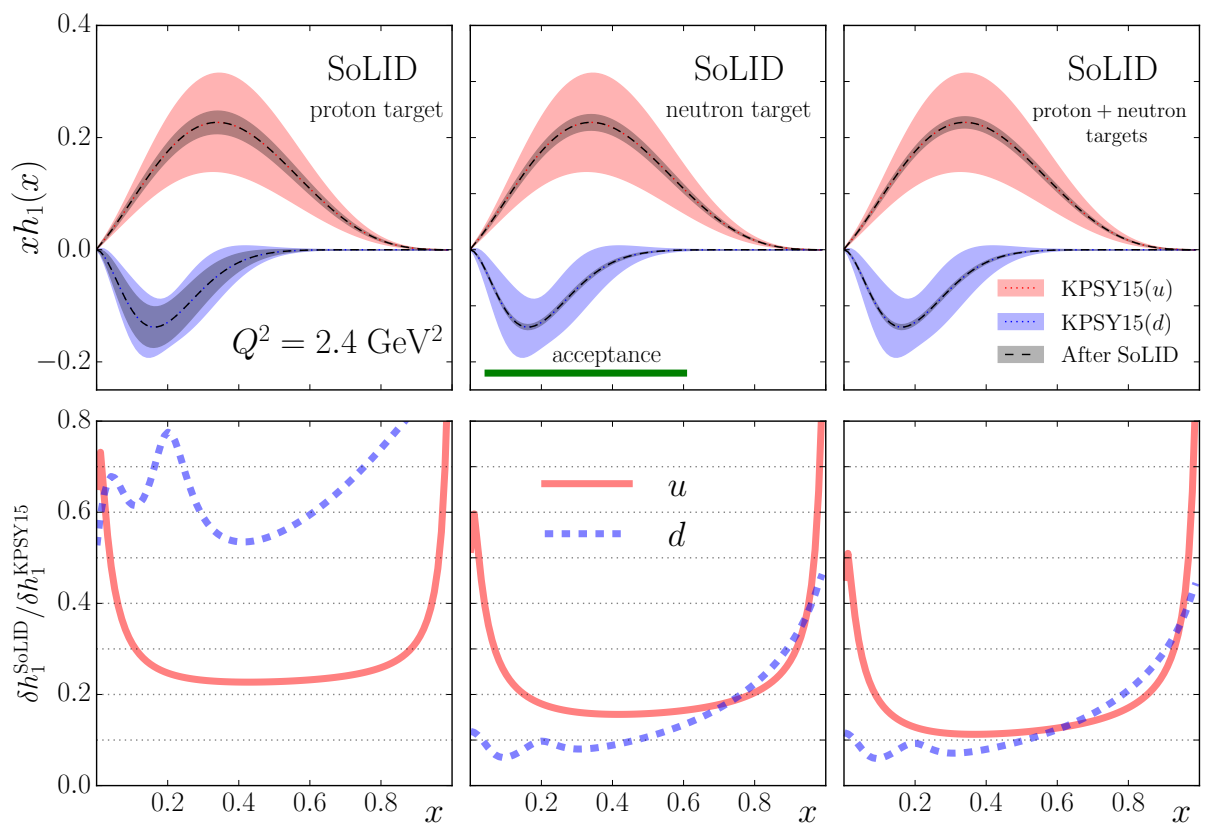

Figure 3: Upper panels: $u$-quark and $d$-quark transversity distributions at $Q^{2}=2.4\left(\mathrm{GeV}^{2}\right)$ as a function of $x$ with existing errors from KPSY15 (light shade area) and the estimated errors after the SoLID data (both statistical and systematical errors are included in quadrature) are taken into account. The acceptance region in $x$ of the SoLID experiment is indicated by the green horizontal line. Left plot: only the proton target data are taken into account, central plot: only the neutron target data are taken into account, right plot: combination of proton and neutron targets data are taken into account. Bottom panels: The ratio of the estimated errors and the current errors of transversity, $\delta h_{1}^{\mathrm{SoLID}} / \delta h_{1}^{\mathrm{KPSY} 15}$, for $u$ (solid line) and $d$ (dashed line) quarks. Left plot: the proton target, central plot: the neutron target, right plot: combination of proton and neutron targets. The "bumps" around $x \simeq 0.2$ of the $d$-quark ratio plots are artifacts of usage of Soffer positivity bound when parametrizing transversity. (The figure is from Ref. [8])

program at JLab $12 \mathrm{GeV}$ will provide unprecedented precision data with a series of well planned experiments using several detectors in multiple halls. As data taking began this year, we are in an exciting time of a new era.

\section{References}

[1] Z.-B. Kang, A. Prokudin, P. Sun, F. Yuan, Phys. Rev. D 93 (2016) 014009.

[2] M. Anselmino, M. Boglione, U. D’Alesio, J. O. Gonzalez Hernandez, S. Melis, F. Murgia and A. Prokudin, Phys. Rev. D 92 (2015) 114023.

[3] M. Radici and A. Bacchetta, Phys. Rev. Lett. 120 (2018) 192001.

[4] H. Gao et al., Eur. Phys. J. Plus 126 (2011) 2.

[5] X. Qian et al. (Jefferson Lab Hall A Collaboration), Phys. Rev. Lett. 107 (2011) 072003.

[6] J. Huang et al. (Jefferson Lab Hall A Collaboration), Phys. Rev. Lett. 108 (2012) 052001.

[7] J. P. Chen et al. (SoLID Collaboration), arXiv:1409.7741 [nucl-ex].

[8] Z. Ye et al., Phys. Lett. B 767 (2017) 91. 